\title{
P-selectin blockade ameliorates lupus nephritis in MRL/Ipr mice through improving renal hypoxia and evaluation using BOLD-MRI
}

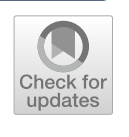

\author{
Liwen Zhang ${ }^{1 \dagger}$, Sheng $\mathrm{Chen}^{2 \dagger}$, Yan $\mathrm{Liu}^{1 \dagger}{ }^{\dagger}$, Xueqin $\mathrm{Xu}^{3}$, Qianying Zhang ${ }^{1}$, Shuxin Shao ${ }^{1}$, Weiming Wang ${ }^{1}$ \\ and Xiao Li ${ }^{1 *}$ (D)
}

\begin{abstract}
Background: Lupus nephritis is one of the most common and severe complications of systemic lupus erythematosus, of which poor prognosis is indicated by aggravated renal hypoxia and tubulointerstitial fibrosis. Cell adhesion molecules play a key role in the progression of lupus nephritis tubulointerstitial lesion, including P-selectin, which mediates the rolling of leukocytes and subsequent adhesion and infiltration and then initiates the inflammatory immune response and ischemia and hypoxia injury. However, the effects and mechanisms of P-selectin in lupus nephritis remain to be investigated, and a noninvasive measurement of lupus nephritis tubulointerstitial hypoxia and fibrosis remains to be explored.
\end{abstract}

Methods: Thirty-four MRL/Ipr mice were randomly divided into the following three groups: MRL/Ipr, saline, and antiP-selectin, which consisted of no treatment, treatment with normal saline, and treatment with anti-P-selectin monoclonal antibody (mAb) from 12 to 16 weeks of age, respectively. Ten male C57BL/6 mice of the same age served as normal controls. 24-h urinary protein, urinary albumin-creatinine ratio, and periodic acid-Schiff were used to assess kidney damage; Western blot or immunohistochemical staining of the hypoxia probe Hypoxyprobe ${ }^{\mathrm{TM}}-1$, hypoxiainducible factor $1 a(H I F-1 a)$, and CD31 were used to evaluate hypoxia in renal tissue; and NADPH oxidase subunit gp91 phox and p22phox were used to examine renal oxidative stress. The correlation between kidney injury and blood oxygen level-dependent magnetic resonance imaging (BOLD-MRI) was calculated to assess the clinical value of BOLD-MRI.

Results: P-selectin is upregulated in lupus nephritis. Blocking P-selectin with mAb alleviated renal tubulointerstitial fibrosis, renal hypoxia, and peritubular capillary loss, without alteration of the levels of lupus activity indicators, antidsDNA antibody, or complement C3. BOLD-MRI showed that the reduced R2* values in the renal cortex and medulla of lupus mice were increased when treated with anti-P-selectin mAb as compared with those treated with normal saline, which were negatively correlated with Hypoxyprobe ${ }^{\mathrm{TM}}-1$ hypoxia probe and the expression of HIF-1a.

Conclusions: Early intervention of lupus nephritis with anti-P-selectin mAb can significantly improve the hypoxic state of the kidney and reduce the severity of tubulointerstitial lesions. BOLD-MRI techniques are noninvasive and

\footnotetext{
*Correspondence: lixiao-rj@163.com

'Liwen Zhang, Sheng Chen and Yan Liu are contributed equally to this work

1 Department of Nephrology, Ruijin Hospital, Shanghai Jiao Tong University School of Medicine, 197 Ruijin Er Road, Shanghai 200025, People's Republic of China

Full list of author information is available at the end of the article
}

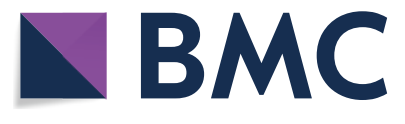

(c) The Author(s) 2020. This article is licensed under a Creative Commons Attribution 4.0 International License, which permits use, sharing, adaptation, distribution and reproduction in any medium or format, as long as you give appropriate credit to the original author(s) and the source, provide a link to the Creative Commons licence, and indicate if changes were made. The images or other third party material in this article are included in the article's Creative Commons licence, unless indicated otherwise in a credit line to the material. If material is not included in the article's Creative Commons licence and your intended use is not permitted by statutory regulation or exceeds the permitted use, you will need to obtain permission directly from the copyright holder. To view a copy of this licence, visit http://creativecommons.org/licenses/by/4.0/. The Creative Commons Public Domain Dedication waiver (http://creativecommons.org/publicdomain/zero/1.0/) applies to the data made available in this article, unless otherwise stated in a credit line to the data. 
can dynamically evaluate the changes in renal lesions and intrarenal oxygenation levels before and after treatment in lupus nephritis.

Keywords: Lupus nephritis, Hypoxic, Tubulointerstitial fibrosis, BOLD-MRI

\section{Background}

Systemic lupus erythematosus (SLE) is a systemic autoimmune disease affecting various organs. Lupus nephritis (LN) is one of the most common and severe complications of SLE, and some cases eventually progress to end-stage renal disease (ESRD). It is reported that renal hypoxia and tubulointerstitial fibrosis are associated with the poor prognosis in LN [1], and cell adhesion molecules play a key role in the progression of LN tubulointerstitial lesion [2, 3]. P-selectin, an adhesion molecule expressed on the surface of activated endothelia and platelets, mediates the rolling of leukocytes and subsequent adhesion and infiltration [4], which initiate the inflammatory immune response and ischemia and hypoxia injury $[5,6]$. However, the effects and mechanisms of P-selectin in glomerulonephritis, especially LN, remain to be explored.

Inflammation caused by immune complex deposition in peritubular capillary (PTC) subendothelium, tubular basement membrane, or interstitium leads to tubulointerstitial lesion and LN progression, and tubulointerstitial hypoxia is a common mechanism for the development of various chronic kidney diseases into ESRD. At present, there is still a lack of ideal noninvasive methodology in the clinical evaluation of LN tubulointerstitial hypoxia or fibrosis. Renal diffusion-weighted imaging (DWI) combined with blood oxygenation level-dependent (BOLD) imaging has been used as a novel magnetic resonance (MR) functional examination technique that reflects changes in renal ischemia, hypoxia, and medullary oxygenation levels [7-9]. Pedersen and colleagues [10] confirmed that the apparent relaxation rate (R2*) value is strongly associated with regional renal oxygen content measured by oxygen-sensitive microelectrodes in pigs and have promoted BOLD magnetic resonance imaging (MRI) as a noninvasive estimate of renal partial oxygen pressure. In previous research, we assessed the utility of BOLD and DWI MRI in discrimination of renal involvement and pathological changes in $65 \mathrm{LN}$ patients and 16 healthy volunteers and found a negative correlation between R2* values of the renal medulla and proteinuria $(r=-0.244, P<0.05)$ as well as the degree of tubulointerstitial lesion $(r=-0.242, P<0.05)$ [11]. However, investigations on the application value of BOLD-MRI in evaluating the damage of LN are still limited [12].

Here, we investigate the pathogenesis of LN tubulointerstitial lesion and demonstrated the renoprotective role of anti-P-selectin monoclonal antibody (mAb) in renal function, pathological characteristics, and tubulointerstitial hypoxia status of an LN animal model (MRL/ lpr mice). Our findings suggest that blocking P-selectin with $\mathrm{mAb}$ alleviates renal tubulointerstitial fibrosis, renal hypoxia, and PTC loss without alteration of the levels of lupus activity indicators, anti-dsDNA antibody, or complement C3. BOLD-MRI showed that the R2* of the renal cortex and medulla of lupus mice treated with anti-Pselectin $\mathrm{mAb}$ was higher than that of the saline control littermates, promoting BOLD-MRI as a proper measurement of renal hypoxia in LN.

\section{Methods}

\section{Animal experiments}

Animal maintenance and experimental procedures were approved by the Animal Care Committee of Ruijin Hospital, Shanghai Jiao Tong University School of Medicine (Shanghai, China). Thirty-four MRL/lpr mice aged 11 weeks were introduced and cultivated by the Shanghai Experimental Animal Center of the Chinese Academy of Sciences from the Jackson Laboratory (USA), and 10 male C57BL/6 mice of the same age served as normal controls. For studies involving the role of anti-P-selectin $\mathrm{mAb}$ in $\mathrm{LN}, \mathrm{MRL} / \mathrm{lpr}$ mice were randomly divided into the following three groups: MRL/lpr, saline, and antiP-selectin groups, which consisted of no treatment, treatment with normal saline, and treatment with antiP-selectin mAb (clone RB40.34, IgG1 $\lambda$; BD Biosciences, San Diego, California, United States) intraperitoneally at $2 \mathrm{mg} / \mathrm{kg}$ body weight twice a week from 12 to 16 weeks of age, respectively. Mice were housed in a specific pathogen-free room at a constant temperature of $22 \pm 2{ }^{\circ} \mathrm{C}$ and a constant humidity of $50 \pm 5 \%$ under a 12 -h day/night cycle. Mice were given free access to chow and water.

\section{Physiologic parameters}

Before the mice were euthanized, they were provided water ad libitum, and 24-h urine was collected in metabolic cages. The urinary albumin concentration was measured using a mouse albumin enzyme-linked immunosorbent assay (ELISA) quantitation set (Bethyl Laboratories, Inc., Montgomery, TX, USA). The urinary creatinine concentration in the same sample was measured using the QuantiChrom ${ }^{\mathrm{TM}}$ Creatinine Assay Kit (BioAssay Systems, Hayward, CA, USA) according to the manufacturer's protocol. The serum was collected 
through the post-glomus venous plexus after anesthetization; anti-dsDNA (FUJIFILM Wako Shibayagi Corporation, Japan) and complement C3 (Icllab, Portland, OR, USA) levels were measured with ELISA kits according to the manufacturer's protocol.

\section{Kidney histopathology}

The kidneys were removed from anesthetized mice and immediately fixed in $4 \%$ paraformaldehyde, embedded in paraffin, and sectioned at $4 \mu \mathrm{m}$. The sections were stained with periodic acid-Schiff (PAS). PAS micrographs were observed by two renal pathologists independently to estimate semi-quantitative scores for glomerular and tubulointerstitial areas according to the area affected by the lesion: 0 (no), $1(\leq 25 \%), 2(25 \%$ to $50 \%), 3(\geq 50 \%)$, in which glomerular $0-9$ points, including mesangial cell proliferation $0-3$ points; mesangial matrix hyperplasia $0-3$ points; sclerosis $0-3$ points; tubulointerstitial $0-9$ points, including renal tubular atrophy $0-3$ points; interstitial fibrosis $0-3$ points; interstitial cells infiltration $0-3$ points.

\section{Immunohistochemistry staining}

One hour before the sacrifice of mice, the hypoxia probe Hypoxyprobe ${ }^{\mathrm{TM}}-1$ (Hypoxyprobe, Inc., Burlington, MA, USA) at $60 \mathrm{mg} / \mathrm{kg}$ body weight was injected intraperitoneally, and the intensity and distribution area were analyzed by immunohistochemical (IHC) staining according to the manufacturer's protocol. IHC staining was performed on paraffin-embedded kidney sections following standard procedures by incubating the sections in a primary antibody against P-selectin (Cell Signaling Technology, Inc., Danvers, MA, USA), hypoxia-inducible factor $1 \alpha$ (HIF- $1 \alpha$; Novus Biologicals, Inc., Littleton, CO, USA), and CD31 (Abcam, Cambridge, MA, USA) at $4{ }^{\circ} \mathrm{C}$ overnight. After washing, the sections were incubated with biotinylated secondary antibodies, followed by incubation with an avidin-biotin-peroxidase complex for DAB substrate development using the ABC kit (Vector Laboratories, Burlingame, CA, USA) at room temperature, and they were mounted using Aqua PolyMount (Polysciences, Inc., Warrington, PA, USA). Images were acquired using a Leica DM1000 microscope with a digital camera. The results of immunohistochemistry were determined by two renal pathologists independently according to the distribution of light staining in renal tissue with a semi-quantitative score of $0-3$, in which 0 indicates negative staining; 1 point, occasional positive staining; 2 points, focal positive staining; and 3 points, diffuse positive staining.

\section{Western blot analysis}

Renal cortical tissues were ground and lysed, and cells were collected and lysed in RIPA buffer containing protease inhibitor cocktail. Equal amounts of protein samples were loaded on sodium dodecyl sulfate polyacrylamide gels, transferred to polyvinylidene difluoride membranes (Millipore, MA, USA), probed with antibodies, and visualized with the Luminescent Imaging Workstation (Tanon, China). The band intensities were quantified using ImageJ. Mouse anti- $\beta$-actin antibody (Sigma, MA, USA) was used as loading control.

\section{Renal BOLD-MRI examination}

Mice were fasted for 4 to $8 \mathrm{~h}$ before BOLD-MRI. After anesthetization, mice were placed in a prone position. The limbs were fixed, and the animal-specific eightchannel high-resolution head coil was placed. The T1WI, T2WI, DWI, and BOLD images of kidney cross section were scanned using a GE Signa Excite 3.0T MR scanner. For cross-sectional T1WI and T2WI imaging, sequences included fast spin echo (FSE) T1WI [repetition time (TR)/echo time (TE) 540/12.7 ms], FSE T2WI (TR/TE $4000 / 46.2 \mathrm{~ms}$ ), layer thickness $2.4 \mathrm{~mm}$, layer spacing $0.2 \mathrm{~mm}$, field of view (FOV) $6 \mathrm{~cm} \times 6 \mathrm{~cm}$, and matrix $256 \times 224$. The scope of the scan included both kidneys. For cross-sectional BOLD imaging, sequences included multiecho fast gradient-echo sequence, 8 gradient echoes, TR $110 \mathrm{~ms}$, TE $2.0-27.5 \mathrm{~ms}$, layer thickness $2 \mathrm{~mm}$, and spacing $0.2 \mathrm{~mm}$, with 5 levels of coronal imaging centered on the renal hilum. Parameters were set as flip angle $20^{\circ}$, bandwidth 125 , number of excitations (NEX) 1 , FOV $15 \mathrm{~cm} \times 15 \mathrm{~cm}$, and matrix $224 \times 192$ (8 frames per layer). The data were transferred to the ADW4.2 workstation, and the $\mathrm{R}^{*}$ values of the renal cortex and medulla were measured by Functool software on the image near the renal hilum. The R2* value measurement method consisted of the following: the window width/ window position of the R2* image was adjusted to about $60.0 / 5.0$, and the R2* color map of the kidney was used to measure the $\mathrm{R}^{*}$ value of the renal cortex. The region of interest (ROI) was posited on the clear boundary of the cortex and medulla manually, and 3 ROIs were placed on each side of the kidney cortex and medulla to calculate their average [11].

\section{Statistical analyses}

The group data were expressed as the mean \pm standard error of the mean (SEM). Comparisons between the two groups were performed using an unpaired $t$ test after determining the distribution and variance of the data. One-way analysis of variance (ANOVA) followed by Tukey's multiple comparisons test was used when more 
than two groups were present. All tests were two-tailed, and $P<0.05$ was considered to be a statistically significant result.

\section{Results}

P-selectin expression in the kidney of MRL/lpr lupus mice

We analyzed the expression level and distribution characteristics of P-selectin in renal tissues of MRL/lpr mice by IHC staining. The renal tissue of C57BL/ 6 mice in the control group expressed P-selectin at a low level, and the positive area of lupus mouse kidney increased as compared with that in the control group, moderately in glomeruli and distinctively in tubulointestitium (Fig. 1a). There was no change in the expression of P-selectin in $\mathrm{MRL} / \mathrm{lpr}$ renal tissue after treatment with normal saline, but anti-P-selectin mAb blocked P-selection expression distinctively (Fig. 1a). Moreover, the expression changes of P-selectin in IHC images in Fig. 1a were confirmed by western blot. It was shown through western blot analysis that renal P-selectin expression was significantly upregulated in MRL/lpr mice compared with that in C57BL/6 mice (Fig. 1b, c), and the expression level of P-selectin in the anti-P-selectin group was significantly decreased compared with that in the normal saline group (Fig. 1d, e).

\section{Anti-P-selectin mAb ameliorated kidney injury in MRL/lpr mice}

To investigate the effect of P-selectin blockade on LN, we evaluated the variation in histopathology and urinary protein and in MRL/lpr mice treated with normal saline or anti-P-selectin mAb. PAS staining micrographs showed severe glomerular, interstitial, and arteriolar lesions in both LN and normal saline-treated mice, which was characterized by mesangial expansion, basement membrane thickening, tubular epithelial

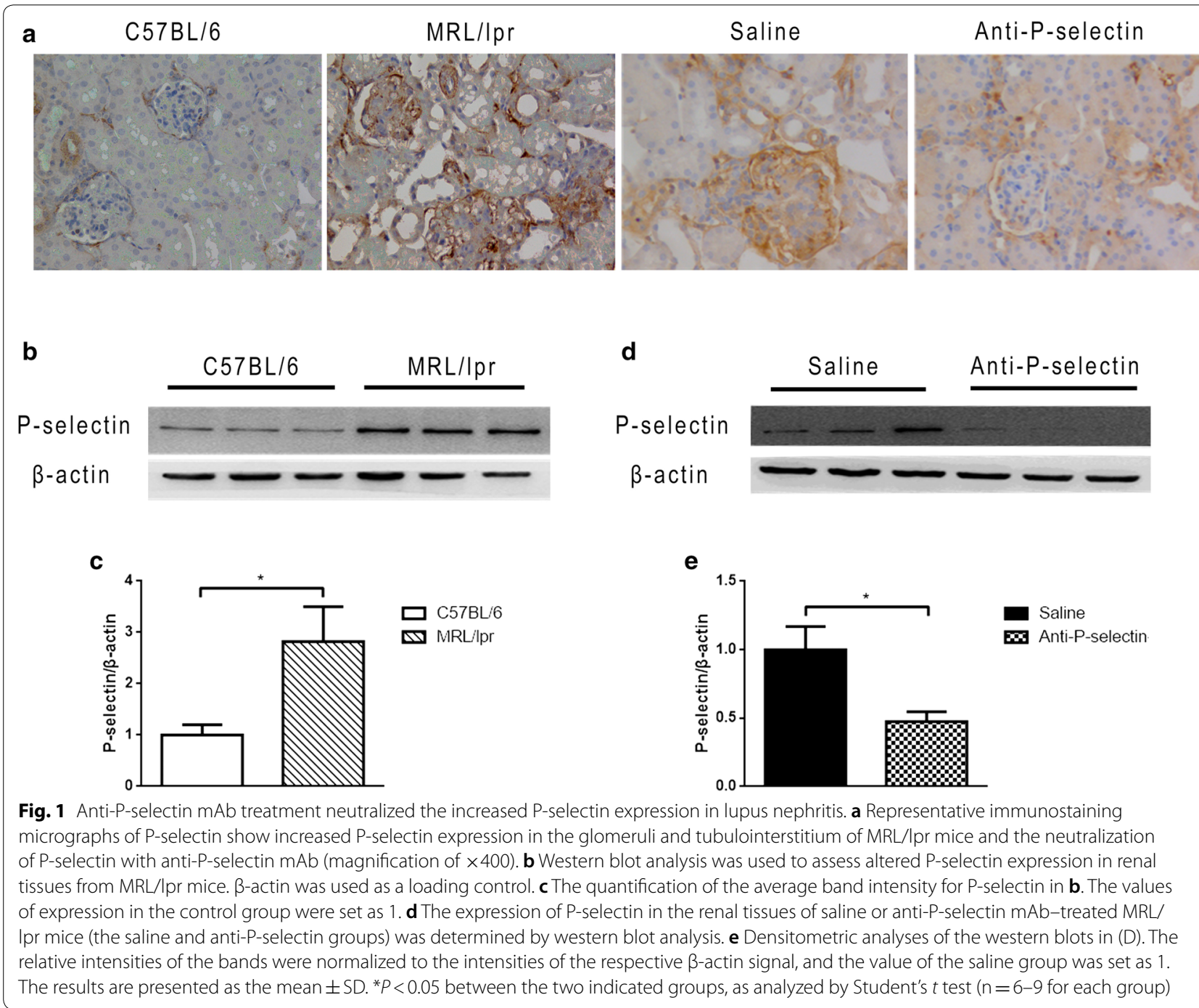


detachment, interstitial inflammatory infiltration, and especially the remarkable accumulation of perivascular inflammatory cells (Fig. 2a). P-selectin blockade in $\mathrm{MRL} / \mathrm{lpr}$ mice prevented these histopathologic lesions to a great extent, which presented as moderate mesangial proliferation, inconspicuous tubule atrophy, and less inflammatory infiltration (Fig. 2a). Moreover, both kidney and tubulointerstitial injury scores in the MRL/lpr group increased significantly as compared with those in the C57BL/6 group, and they decreased significantly in the anti-P-selectin group as compared with those in the saline group (Fig. 2b), indicating that anti-P-selectin $\mathrm{mAb}$ have a significant protective effect on LN injury.

The urinary albumin/creatinine ratio (ACR) of MRL/lpr mice was significantly higher than that of $\mathrm{C} 57 \mathrm{BL} / 6$ mice at 12 weeks of age (Fig. 2c). We then evaluated the urinary protein levels and variation in MRL/lpr mice treated with normal saline or anti-P-selectin mAb (Table 1). After 4 weeks of anti-P-selectin mAb treatment (A2 group), the urinary ACR declined significantly compared with that of 12-week-old MRL/lpr mice ( ${ }^{*} P=0.048$ ); however, the urinary ACR did not show a difference after treatment with normal saline for 4 weeks $(P=0.22$; A1 group). In addition, at 16 weeks of age, the 24-h urine protein level in group A2 was significantly lower than that in group A1 $\left({ }^{\#} P=0.02\right)$. We further investigated the effect of P-selectin blockade on urinary protein at different phases of LN, including from 12 to 20 weeks of age (P-selectin blockade and normal saline treatment as the B2 and B1 groups, respectively) and 16 to 20 weeks of
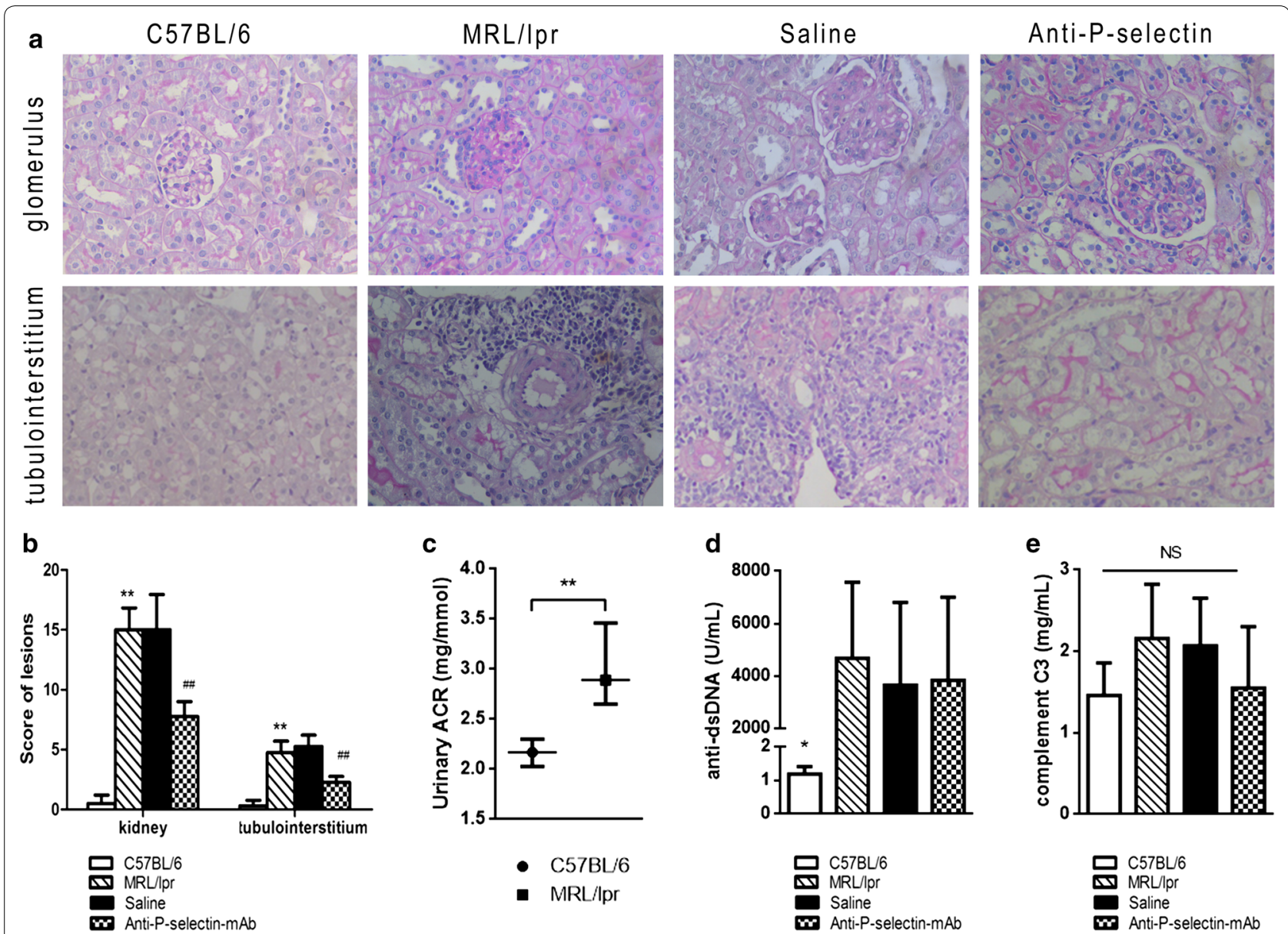

Fig. 2 Anti-P-selectin mAb treatment ameliorated kidney injury in MRL/lpr mice. a Representative PAS staining micrographs of the glomerulus and tubulointerstitium in the C57BL/6, MRL/lpr, saline, and anti-P-selectin groups (magnification of $\times 400$ ). $\mathbf{b}$ Semi-quantitative score of lesions in terms of kidney and tubulointerstitium. ${ }^{*} P<0.01$ compared with $C 57 \mathrm{BL} / 6$ group; ${ }^{\#} P<0.01$ compared with saline group. c Urinary albumin-creatinine ratio of the MRL/Ipr group compared with the C57BL/6 group at the age of 12 weeks. The results are presented as the medium (first quartile, third quartile). ${ }^{* *} P<0.01$ between the indicated two groups $\left(n=10-13\right.$ for each group). $\mathbf{d}$ Serum anti-dsDNA levels of each group. ${ }^{*} P<0.05$ compared with the MRL/Ipr, saline, and anti-P-selection groups as analyzed by ANOVA followed by Tukey's multiple comparisons test. e Complement C3 levels of each group. No statistical significance among groups 
Table 1 Urinary protein alteration in MRL/lpr mice with normal saline or anti-P-selectin monoclonal antibody treatment

\begin{tabular}{|c|c|c|c|c|c|c|c|c|c|}
\hline & \multicolumn{3}{|c|}{ 24-h urinary protein $(\mathrm{mg})$} & \multicolumn{3}{|c|}{ Urinary protein concentration $(\mathrm{mg} / \mathrm{mL})$} & \multicolumn{3}{|c|}{ Urinary ACR (mg/mmol) } \\
\hline & 12 wks & 16 wks & 20 wks & 12 wks & 16 wks & 20 wks & 12 wks & 16 wks & 20 wks \\
\hline \multicolumn{10}{|c|}{ Norma saline treatment } \\
\hline $\mathrm{A} 1$ & $3.24 \pm 1.47$ & $4.22 \pm 1.71$ & - & $1.60 \pm 0.82$ & $2.70 \pm 1.38^{* *}$ & - & $4.36 \pm 3.19$ & $5.78 \pm 4.43$ & - \\
\hline B1 & $3.0 \pm 1.71$ & $4.08 \pm 1.83$ & $4.2 \pm 5.95$ & $1.63 \pm 1.02$ & $2.50 \pm 1.39$ & $2.79 \pm 1.89$ & $3.39(2.74,6.32)$ & $3.34(2.78,6.74)$ & $5.02(2.8,33.77)$ \\
\hline \multicolumn{10}{|c|}{ Anti-P-selectin mAb treatment } \\
\hline A2 & $1.83 \pm 0.83$ & $1.19 \pm 0.58^{\#}$ & - & $1.57 \pm 0.60$ & $2.56 \pm 1.55$ & - & $2.69 \pm 0.45$ & $1.14 \pm 0.10^{*}$ & - \\
\hline B2 & $3.67 \pm 2.22$ & $1.69 \pm 0.89^{\# \#}$ & $2.14 \pm 1.41 \boldsymbol{\Delta}$ & $1.94 \pm 0.82$ & $2.16 \pm 0.80$ & $3.30 \pm 1.06$ & $2.36(2.14,3.11)$ & $2.68(1.24,3.21)$ & $2.35(2.0,8.65) \boldsymbol{\Lambda}$ \\
\hline $\mathrm{C} 2$ & - & $3.75 \pm 1.99$ & $3.94 \pm 3.17$ & - & $2.80 \pm 0.47$ & $3.58 \pm 0.74$ & - & $2.35(2.0,8.65)$ & $10.07(2.54,12.59)$ \\
\hline
\end{tabular}

The results are presented as the mean \pm SD or median (first quartile, third quartile). Saline groups:A1 and B1 groups were treated with normal saline from 12 to 16 weeks and from 12 to 20 weeks, respectively. Anti-P-selectin groups: A2, B2, and C2 groups were treated with anti-P-selectin mAb from 12 to 16 weeks, from 12 to 20 weeks, and from 16 to 20 weeks, respectively

Compared with the baseline (that in 12 weeks for the same group), ${ }^{*}, * P<0.05$. Compared with the same age saline group,,$\# \#<0.05$. Compared with the $C 2$ group, $\boldsymbol{\Delta}_{P<0.05}$

age (P-selectin blockade treatment as the $\mathrm{C} 1$ group). The ACR of the B2 group (MRL/lpr mice treated with anti$\mathrm{P}$-selectin $\mathrm{mAb}$ at $12-20$ weeks of age) was moderately lower than that of the normal saline control B1 group without statistical significance. Similar to the results in the A groups, the 24-h urinary protein in the B2 group at the age of 16 weeks was significantly lower than that in the B1 group ${ }^{\# \#} P=0.019$ ), but there was no significant difference between the two groups at 20 weeks. Moreover, the 24-h urinary protein and ACR at 20 weeks in the B2 group were significantly lower than that in the $\mathrm{C} 2$ group that was treated with anti-P-selectin $\mathrm{mAb}$ from 16

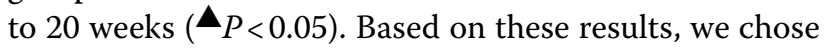
$\mathrm{MRL} / \mathrm{lpr}$ mice aged 12 weeks with 4 weeks of P-selectin blockade for the subsequent research.

We also detected anti-double-stranded DNA (dsDNA) antibody and complement $\mathrm{C} 3$ levels in serum to investigate the system activity of SLE in mice. The level of antidsDNA antibody in LN mice increased sharply (by about 5000-fold) as compared with that in the C57BL/6 control mice, but there was no significant difference in the level of anti-dsDNA antibody between the saline and anti-Pselectin mAb-treated groups (Fig. 2d). Complement C3 levels among the four groups were moderately various
(Fig. 2e), as well as between different weeks of age (data not provided). These results demonstrated that P-selectin blockade protected renal injury in MRL/lpr LN mice without affecting the systemic SLE activity.

\section{Anti-P-selectin mAb attenuated renal hypoxia in MRL/lpr mice}

We employed immunohistochemistry to detect the distribution of Hypoxyprobe ${ }^{\mathrm{TM}}-1$ hypoxic probe in renal tissue. It showed mild positive expression in the kidney of C57BL/6 mice; however, it was widely distributed in the renal tissue of $\mathrm{MRL} / \mathrm{lpr}$ mice without intervention, including glomerular mesangial area, tubular epithelial cells, and interstitial and perivascular areas, suggesting severe hypoxia in MRL/lpr kidney. The positive area of Hypoxyprobe ${ }^{\mathrm{TM}}-1$ hypoxia probe in the kidney of the anti-P-selectin group declined as compared with that in the saline group (Fig. 3a). Moreover, HIF-1 $\alpha$ was wildly expressed in the kidney, and the immunohistochemistry staining image in the C57BL/6 group showed moderately positive expression, but the positive area increased obviously in the MRL/lpr group and more distinctively in the interstitial and medulla area than in the glomeruli (Fig. 3b). There was no significant difference in HIF-1 $\alpha$

\footnotetext{
(See figure on next page.)

Fig. 3 Anti-P-selectin mAb treatment ameliorated renal hypoxia in MRL/lpr mice. a Representative hypoxia probe in situ staining micrographs of by hypoxyprobe ${ }^{\mathrm{TM}}-1$ (magnification of $\times 400$ ). $\mathbf{b}$ Immunohistochemistry staining of HIF-1a in the glomerulus and tubular interstitium (magnification of $\times 400$ ). $\mathbf{c}$ Western blot analysis was used to assess altered HIF-1a expression in renal tissues from MRL/Ipr mice. $\beta$-actin was used as a loading control. $\mathbf{d}$ The quantification of the average band intensity for HIF-1a in $\mathbf{c}$. The values of expression in the control group were set as 1. e The expression of HIF-1a in the renal tissues of saline or anti-P-selectin mAb-treated MRL/lpr mice (the saline and anti-P-selectin groups) was determined by western blot analysis. $\mathbf{f}$ Densitometric analyses of the western blots in $\mathbf{e}$. The relative intensities of the bands were normalized to the intensities of the respective $\beta$-actin signal, and the value of the saline group was set as 1 . The results are presented as the mean \pm SD. ${ }^{*}<0.05$ between the two indicated groups, as analyzed by Student's $t$ test ( $n=6-9$ for each group). $\mathbf{g}$ Peritubular capillary count (PTC) in the kidney of each group. ${ }^{* *} P<0.001$ as analyzed by ANOVA followed by Tukey's multiple comparisons test. $\mathbf{h}$ Immunohistochemistry staining of CD31 for PTC counting in the glomerulus and tubular interstitium (magnification of $\times 400$ )
} 
a

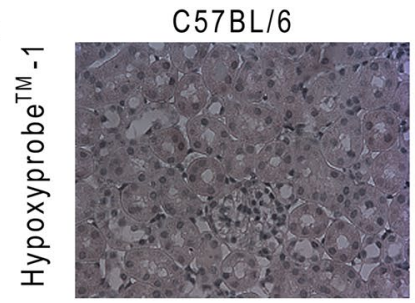

b
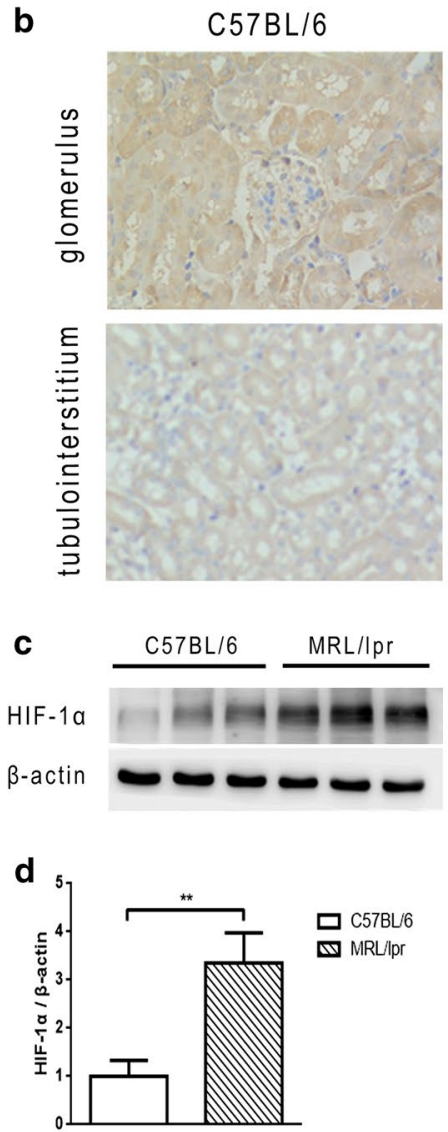

h
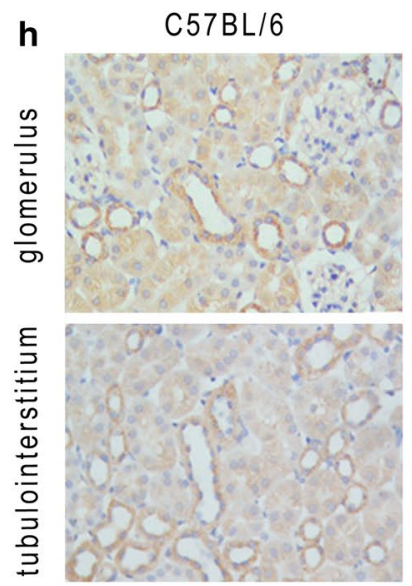
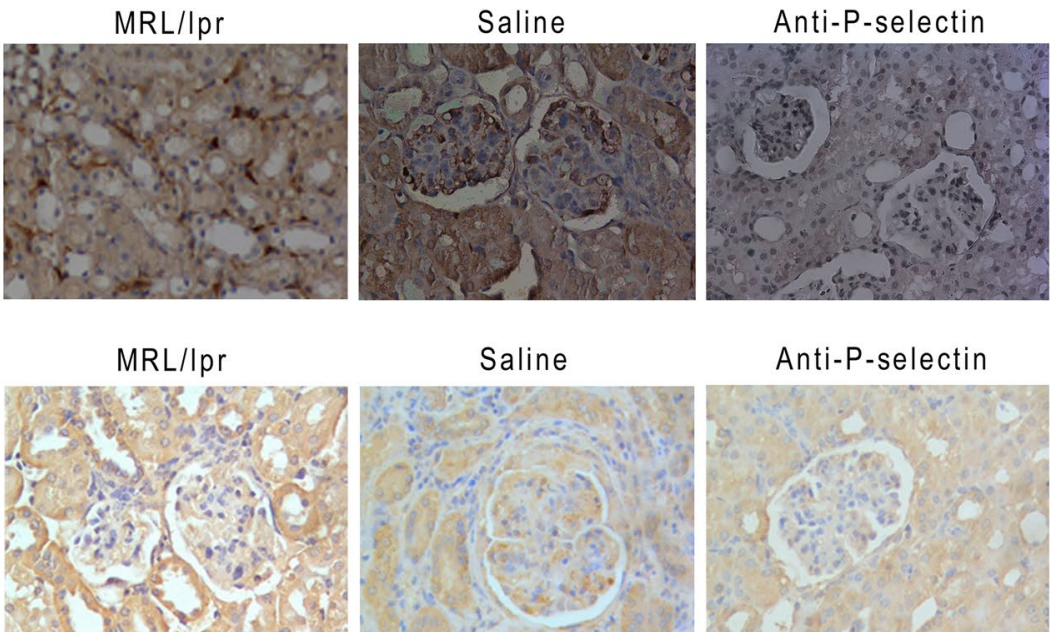

Saline

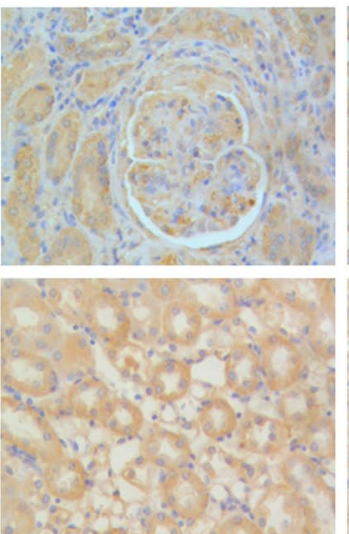

Anti-P-selectin
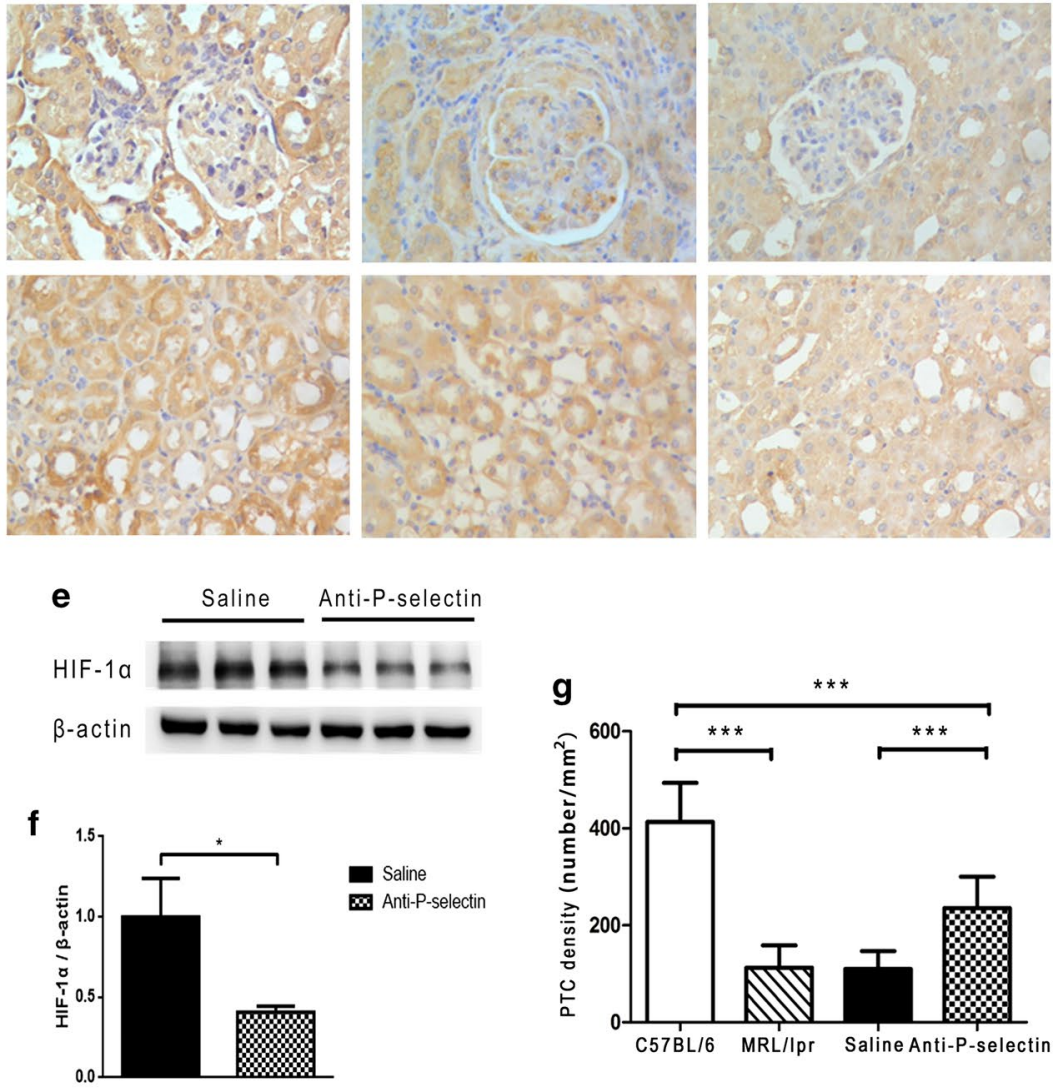

MRL/Ipr

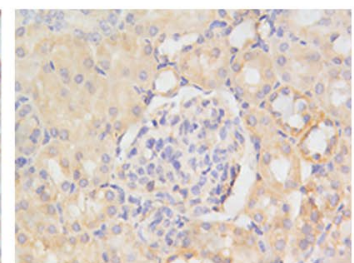

Saline

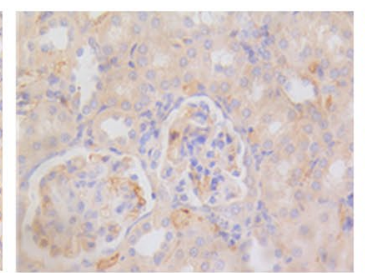

Anti-P-selectin

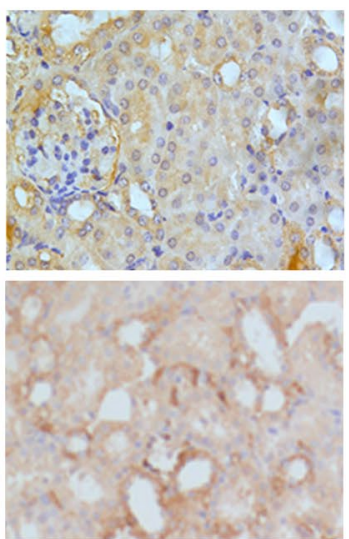


expression between the saline group and the untreated $\mathrm{MRL} / \mathrm{lpr}$ group, but that in the anti-P-selectin $\mathrm{mAb}$ intervention group was restored (Fig. 3b). Furthermore, we confirmed the variation in HIF-1 $\alpha$ and the statistical significance among the groups in protein levels via western blot (Fig. 3c-f). Then, the distribution of capillary was determined with immunohistochemistry staining of CD31, an endothelial marker, and we found that the PTC count in the kidney of the MRL/lpr group reduced significantly as compared with that of the C57BL/6 group (Fig. 3g, h). After the intervention with anti-P-selectin $\mathrm{mAb}$, the PTC count of renal tissue in lupus mice was significantly higher than that in lupus mice treated with normal saline (Fig. 3g, h).
In addition, the distribution of NADPH oxidase subunit gp91phox in mouse kidney was detected by immunohistochemistry to further assess renal oxidative stress. There was no obvious expression in C57BL/6 mouse kidney tissue (Fig. 4a, Line 1), while strong positive staining was detected in the mesangial and tubulointerstitial area of MRL/lpr mice (Fig. 4a, Line 2), and anti-P-selectin $\mathrm{mAb}$ treatment reversed the upregulation of gp91phox in MRL/lpr kidney (Fig. 4a, Line 3 and 4). The expression levels of NADPH oxidase subunits gp91phox and p22phox in MRL/lpr mice were significantly upregulated as evaluated by western blot (Fig. 4b, c), and they decreased after treatment with anti-P-selectin $\mathrm{mAb}$ (Fig. 4a, d, e).

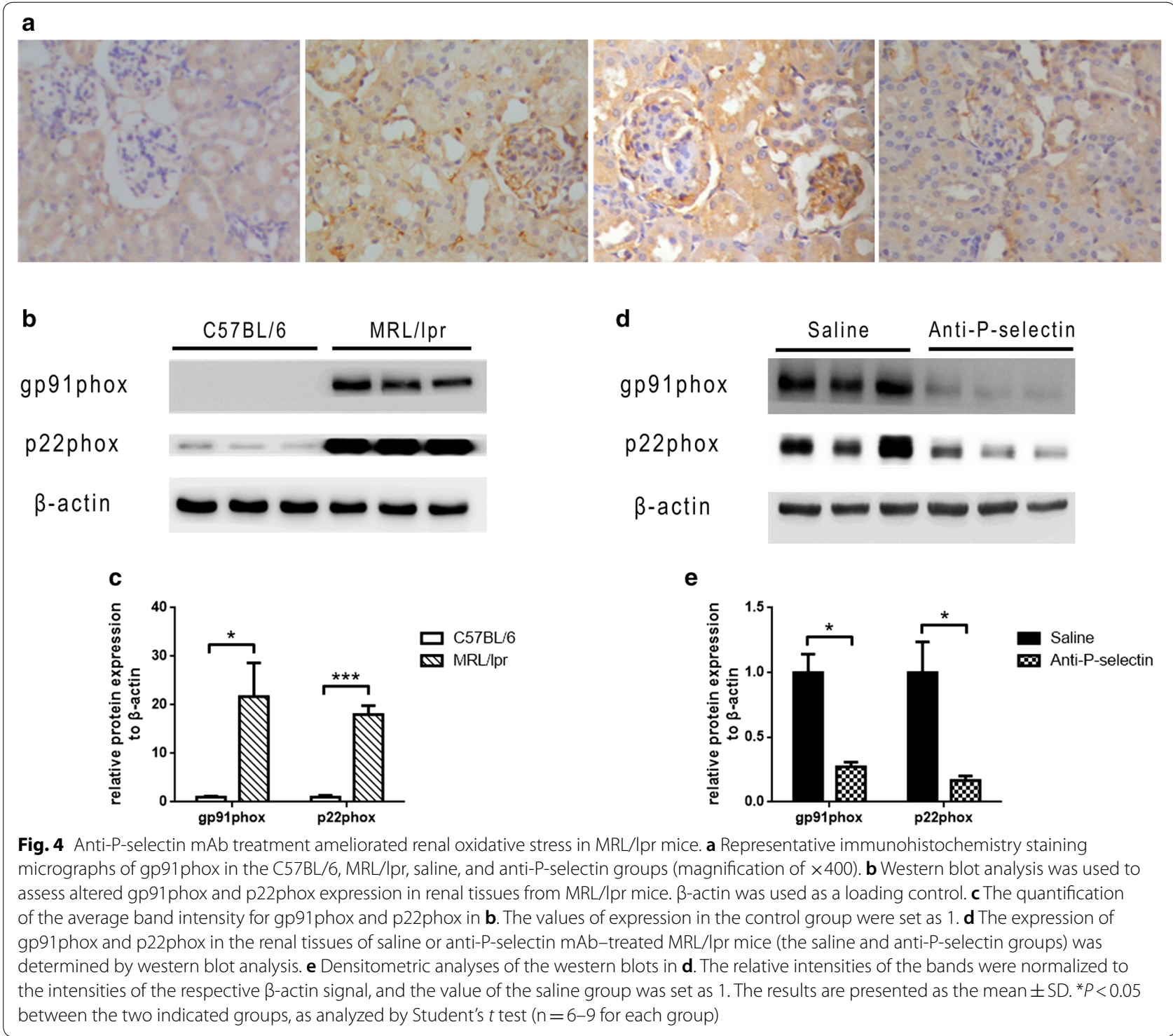




\section{Evaluation of renal hypoxia in MRL/lpr mice through BOLD-MRI}

The R2* image showed that the cortex and medulla in C57BL/6 mouse kidney was clearly defined, and the color of the kidney from the cortex to the medulla was gradually altered from blue to green and then to yellow when comparing the $\mathrm{R} 2$ * color images to the $\mathrm{T} 2$ * gray images (Fig. 5a, b). In the LN and saline groups, because of the abnormal enlargement of the abdominal lymph nodes, the kidney was squeezed to disposition and the structure blurred between the cortex and medulla (Fig. 5a, Line 2) as compared with that in the R2* color map, in which the whole kidney was almost covered by blue (Fig. 5b, Line 2). The normal saline intervention had no obvious effect on the renal R2* map of MRL/lpr mice, while P-selectin blockade recovered the clear boundary between the renal cortex and medulla in MRL/lpr mice (Fig. 5b, Line 3 and 4). The renal cortical and medulla R2* values of MRL/lpr mice were lower than those of normal control C57BL/6 mice, and the anti-P-selectin mAb intervention induced the R2* values of renal cortex and medulla of MRL/lpr mice recovery (Table 2 ).

In the C56BL/6 and MRL/lpr groups, medullary apparent diffusion coefficient (ADC) values were negatively correlated with urinary ACR when $b=500 \mathrm{~s} /$ $\mathrm{mm}^{2}$ (Fig. $5 \mathrm{c}$ ) or $800 \mathrm{~s} / \mathrm{mm}^{2}$ (Fig. 5d) but uncorrelated when $b=200 \mathrm{~s} / \mathrm{mm}^{2}$. The medullary R2* value of MRL/ lpr mice was negatively correlated with the tubulointerstitial lesion score (Fig. 5e) and was also significantly negatively correlated with the expression of Hypoxyprobe $^{\mathrm{TM}}-1$ hypoxia probe (Fig. 5f) or HIF-1 $\alpha$ (Fig. 5g) in glomeruli, tubules, interstitium, and arterioles. Similarly to the former two groups, in LN mice treated with saline or anti-P-selectin $\mathrm{mAb}$, the mean medullary R2* values of the kidney were significantly negatively correlated with the expression of Hypoxyprobe ${ }^{\mathrm{TM}}-1$ hypoxia probe in glomeruli, tubules, interstitium, and arterioles (Fig. 5h) and were also significantly negatively correlated with the expression of HIF- $1 \alpha$ in tubules, interstitium, and arterioles but not glomeruli (Fig. 5i).

\section{Discussion}

Previous studies on LN have primarily focused on glomerular damage, but in recent years, tubulointerstitial lesions have been found to determine the renal prognosis of patients with LN [13]. The most important factor affecting tubulointerstitial lesions is chronic hypoxia, which leads to metabolism disorders, thereby inducing inflammatory reactions and reactive oxygen species (ROS) and causing or aggravating structural and functional damage of tubular epithelial cells and renal pathological changes $[14,15]$. In the process of tubulointerstitial lesions of LN, the deposition of immune complexes in the renal tubular basement membrane and $\mathrm{T}$ cell-mediated immune damage may lead to endothelial cell activation, tissue ischemia and hypoxia, and massive production of ROS in the kidney. Neutrophils adhered and aggregated under the co-affection of ROS, P-selectin, and mitogen-activated protein kinase and accelerated tubulointerstitial damage $[5,16]$. When renal tubular cells encounter hypoxia, fatty acid oxidation is impaired, and glycolysis becomes the main energy source. Due to these metabolic restrictions, the proximal tubular epithelial cells undergo the phenotypic transformation, including cytoskeletal rearrangement and extracellular matrix protein synthesis [17].

Previous studies have shown that MRL/lpr mice might be an appropriate model for studying LN, because they have a good consistency with the process of human LN. In our study, they showed elevated serum anti-ds-DNA antibody levels and proteinuria compared with C57BL/6 mice but no difference in serum complement $\mathrm{C} 3$ and creatinine levels (data not provided). The abnormality of the immune index in the MRL/lpr mouse model is mainly characterized by high-titer anti-nuclear antibody and anti-DNA antibody, but the decrease of complement is not obvious [13]. In fact, we raised MRL/lpr mice to 20 weeks of age, and serum creatinine levels remained unchanged from 12 weeks of age. Passwell et al. [18] showed that slight serum creatinine elevation could be detected in the MRL/lpr strain mice until 22 weeks of age, although the average life span was only 5 months in

\footnotetext{
(See figure on next page.)

Fig. 5 Evaluation of renal hypoxia in MRL/lpr mice through BOLD-MRI. a Representative T2* images of C57BL/6 and MRL/Ipr mice. b Representative R2* images in the C57BL/6, MRL/lpr, saline, and anti-P-selectin groups. c Correlation between medullary ADC and urinary ACR in the C56BL/6 and MRL/Ipr groups when $b=500 \mathrm{~s} / \mathrm{mm}^{2}$. $\mathbf{d}$ Correlation between medullary ADC and urinary ACR in the C56BL/6 and MRL/lpr groups when $b=800 \mathrm{~s} /$ $\mathrm{mm}^{2}$. e Correlation between medullary R2* and tubulointerstitial scores in the C56BL/6 and MRL/lpr groups. $\mathbf{f}$ Correlation between medullary R2* and hypoxic probe score of glomeruli, tubules, interstitium, and arterioles, respectively, in the C56BL/6 and MRL/lpr groups. $\mathbf{g}$ Correlation between medullary R2* and HIF-1a score of glomeruli, tubules, interstitium, and arterioles, respectively, in the C56BL/6 and MRL/lpr groups. $\mathbf{h}$ Correlation between medullary R2* and hypoxic probe score of glomeruli, tubules, interstitium, and arterioles, respectively, in the saline and anti-P-selectin groups. g Correlation between the medullary R2* and HIF-1a score of glomeruli, tubules, interstitium, and arterioles, respectively, in the saline and anti-P-selectin groups
} 

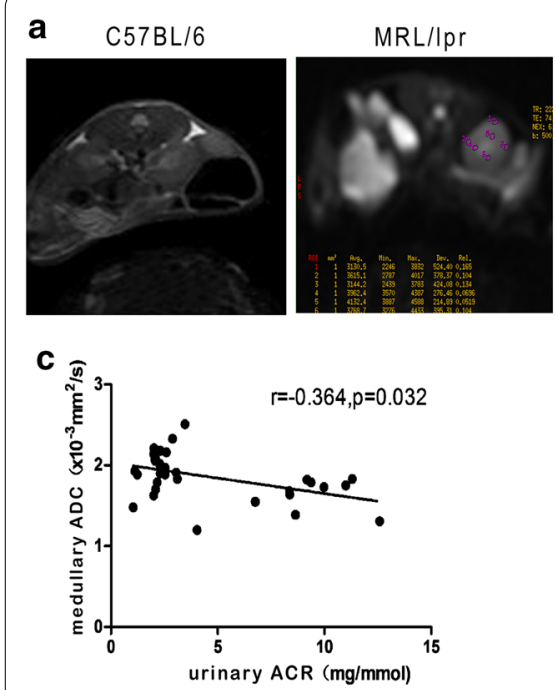

f

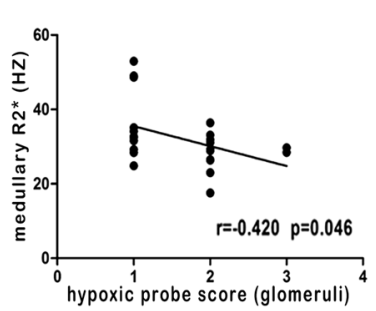

9
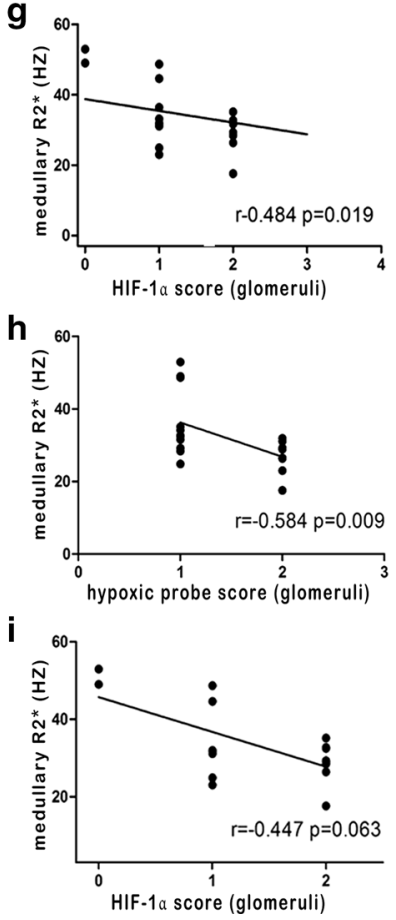

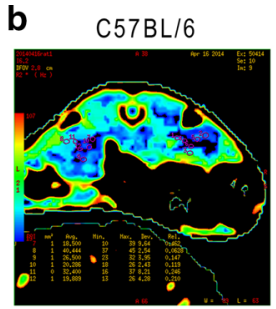

\section{d}

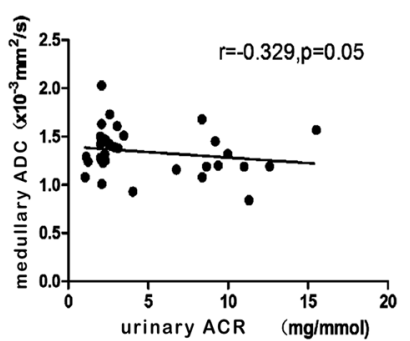

MRL//pr
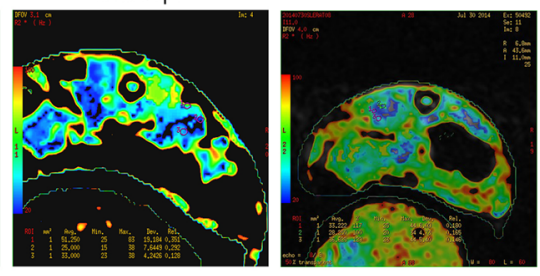

e

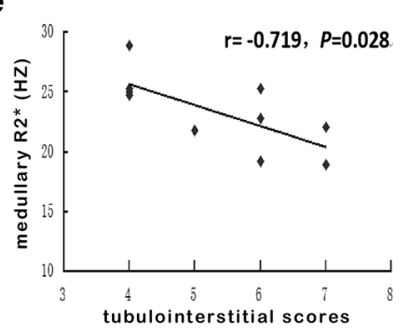

Anti-P-selectin

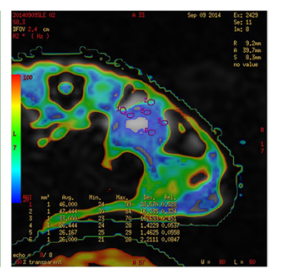

\section{.}


Table 2 Cortical and medullary R2* values of kidneys in mice

\begin{tabular}{lll}
\hline & Cortical R2* value (1/s) & Medullary R2* value (1/s) \\
\hline C57BL/6 & $39.80 \pm 4.30^{*}$ & $28.94 \pm 0.77^{*}$ \\
MRL/lpr & $30.96 \pm 4.54$ & $23.31 \pm 2.16$ \\
Norma saline treatment & $28.52 \pm 2.59$ & $23.17 \pm 2.96$ \\
Anti-P-selectin mAb treatment & $46.37 \pm 9.7^{* *, \# \#}$ & $30.16 \pm 4.26^{*, \#}$ \\
\hline
\end{tabular}

${ }^{*} P<0.05,{ }^{*} P<0.01$ compared with the untreated MRL/lpr group. ${ }^{\#} P<0.05,{ }^{\# \#} P<0.01$ compared with the same age saline group

Cell adhesion molecules are cell-membrane proteins that maintain cell-cell and cell-substrate adhesion and transduce intracellular signaling in some cases. Adhesion molecules are generally divided into five groups: integrins, selectins, cadherins, members of the immunoglobulin superfamily (IgSF) including nectins and others such as mucins [21]. In the kidney, integrins and cadherins are essential for maintaining the epithelial polarity and barrier integrity, while some members of the IgSF and the selectins (ICAM-1, VCAM-1, E-selectin and P-selectin) are essential mediators of both immune and inflammatory responses. P-selectin is a major member of the cell adhesion molecule family, and its role in various glomerular diseases has also received extensive attention [22-24]. P-selctin participates in trafficking of cells of the innate immune system, $\mathrm{T}$ lymphocytes and platelets and is involved in constitutive lymphocyte homing and in chronic and acute inflammation processes, including bacterial infections, glomerulonephritis and SLE [25]. Its expression is significantly upregulated in plasma and renal tissues of patients with LN. P-selectin and its mediated infiltration of dendritic cells and accumulation in renal interstitium initiated a tubulointerstitial inflammatory immunoreaction and histopathological damage in the early stage of LN [26, 27]. However, He et al. [28] found that P-selectin knockout in MRL/lpr mice was not protective but rather accelerated glomerulonephritis with upregulated expression of the chemokine CCL2 in both kidney tissue and in purified renal endothelial cells. In this research, we found that the expression of P-selectin in the MRL/lpr mouse kidney tissue, especially the tubulointerstitium, increased as compared with that in C57BL/6 mice, and the expression was more significant as the degree of renal lesions increased. P-selectin blockade decreased the urinary protein levels in MRL/lpr mice, which was more significant in mice who underwent a 4-week intervention that commenced from 12 weeks of age as compared with 16 weeks of age. In addition, we noted improved renal tubular necrosis and inflammatory cell massive accumulation in the interstitium and pericapillary. We also found that anti-P-selectin mAb had no direct effect on anti-ds-DNA antibody or complement C3 in LN mice, suggesting that the immune mechanisms of P-selectin on $\mathrm{LN}$ progression involve infiltration and differentiation of $\mathrm{T}$ cells and neutrophils rather than B-cell antibody secretion or the complement activation system.

Under normal conditions, the oxygen partial pressure of the renal cortex (about $50 \mathrm{mmHg}$ ) is significantly higher than that in the medulla $(10-20 \mathrm{mmHg})$, causing the medulla to become sensitive to hypoxia and severe tubulointerstitial lesions during stress $[29,30]$. The interstitial blood supply derives from postglomerular blood vessels; thus, glomerular hypertension and sclerosis can cause interstitial ischemia, while the interstitial oxidative stress due to inflammation aggravates hypoxia [1]. We employed the Hypoxyprobe ${ }^{\mathrm{TM}}-1$ hypoxia probe and hypoxia marker HIF-1 $\alpha$ to evaluate renal hypoxia in LN mice and found that their distribution were consistent with a more positive expression in the medulla than that in the cortex, suggesting that there is diffuse hypoxia in the medulla of LN model mice. Hypoxyprobe ${ }^{\mathrm{TM}}-1$ is a hypoxia marker of cells or tissues, and HIF- $1 \alpha$ reflects the ratio of local oxygen supplement to demand. The HIF- $1 \alpha$ pathway is activated when the demand for oxygen is greater than oxygen supplement [31, 32]. In severe tubulointerstitial lesions, the expression of the hypoxia probe and HIF-1 $\alpha$ was upregulated in renal tissues, suggesting that renal hypoxia plays an important role in the development of LN tubulointerstitial lesions. However, P-selectin blockade recovered the expression of hypoxia probe Hypoxyprobe ${ }^{\mathrm{TM}}-1$ and HIF- $1 \alpha$, indicating that LN renal hypoxia was significantly reduced after inhibition of P-selectin. Changes in the expression of NADPH oxidase subunits gp91phox and p22phox suggested that oxidative stress was also involved in this process. Based on these findings, it is speculated that in the early stage of $\mathrm{LN}, \mathrm{P}$-selectin is upregulated in renal tissue, mediating rolling of leukocytes and adhesion to vascular endothelial cells. On one hand, leukocytes cross the blood vessels to invade the interstitial space, causing interstitial inflammation and injury to vascular endothelial cells. On the other hand, the density of capillaries in the renal medulla decreases with endothelial cell injury, so that their supplied oxygen and nutrients are greatly reduced, causing hypoxia and tubulointerstitial lesions in the kidney. 
Hypoxia stimulates endothelial cells to express P-selectin, forming a vicious circle. This suggests that the improvement of tubulointerstitial hypoxia may be the mechanism by which P-selectin monoclonal antibody relieves renal tubulointerstitial fibrosis. Blocking early P-selectin-mediated leukocyte adhesion may have therapeutic implications in improving the prognosis of LN. At present, the human monoclonal antibody against P-selectin, inclacumab, has passed phase II clinical trials in coronary interventions [33, 34], and our findings reveal its potential for the treatment of $\mathrm{LN}$.

In this study, BOLD-MRI showed that the R2* value of the mouse kidney medulla was negatively correlated with the urinary protein level, suggesting that when there is a large amount of proteinuria during glomerular lesions, it is often accompanied by insufficient renal blood perfusion, which can lead to renal tissue, the medulla in particular, ischemia and hypoxia, causing a decrease in the medulla R2* value. In the LN model mice, the renal medulla $\mathrm{R}^{*}$ value was lower than the cortical $\mathrm{R} 2$ * value, which was consistent with the distribution of the hypoxia probe and HIF- $1 \alpha$ in the kidney, indicating that BOLD-MRI can noninvasively evaluate the intrarenal oxygenation level to assess tubulointerstitial damage and prognosis. The blurred boundary between the renal cortex and medulla was recovered in MRL/lpr mice after $\mathrm{P}$-selectin blockade, and the R2* color map reflected the improvement of the hypoxic state in the kidney, after which the metabolism of the kidney cells improved and the oxygen demand increased, so that the $\mathrm{R} 2^{*}$ value of the cortex and medulla increased. In addition, there was no significant correlation between the cortex medulla ADC or R2* values and serum anti-ds-DNA antibody or complement C3 levels, suggesting that immune factors have no direct effect on renal ADC values or R2* values.

\section{Conclusions}

This study showed that P-selectin is upregulated in LN kidney tissue and mediates endothelial-leukocyte adhesion, which in turn causes vascular endothelial injury, intrarenal hypoxia, and inflammatory cell infiltration and plays an important role in LN tubulointerstitial lesions. Early intervention of LN with anti-P-selectin mAb can significantly improve the hypoxic state of the kidney and reduce the severity of tubulointerstitial lesions, providing a new idea for the diagnosis and treatment of $\mathrm{LN}$ clinically. Functional MR DWI and BOLD-MRI techniques are noninvasive and can dynamically evaluate the changes in renal lesions and intrarenal oxygenation levels before and after treatment in LN, which provides potential clinical value.

\section{Abbreviations}

LN: Lupus nephritis; DWI: Diffusion-weighted imaging; BOLD: Blood oxygen level dependent; MR: Magnetic resonance; SE-EPI: Spin echo-echo planar imaging; mGRE: Multiple gradient-recalled-echo; TR: Repetition time; TE: Echo time; ADC: Apparent diffusion coefficient; ROI: Region of interest; Al: Activity index; Cl: Chronicity index; CR: Complete remission; NR: No response; ESRD: End-stage renal disease.

\section{Acknowledgements}

Not applicable.

\section{Authors' contributions}

LZ contributed to data collection, analysis, and interpretation and wrote the manuscript; SC and LY performed the mouse experiments and analysis; XX contributed to the MRI experiment; QZ contributed to statistical analysis; SS contributed to data collection; WW reviewed and revised the manuscript; XL conceived and designed the experiments, contributed to mouse experiments, discussed, reviewed, and revised the manuscript. All authors read and approved the final manuscript.

\section{Funding}

The present study was supported by the National Natural Science Foundation of China (Grant No. 81170671, 81870492), and the National Key Research and Development Program of China (Grant No. 2016YFC1305402).

Availability of data and materials

All data generated or analyzed during this study are included in this article.

\section{Ethics approval and consent to participate}

Animal maintenance and experimental procedures were approved by the Animal Care Committee of Ruijin Hospital, Shanghai Jiao Tong University School of Medicine (Shanghai, China).

\section{Consent for publication}

Not applicable.

\section{Competing interests}

The authors declare that they have no competing interests.

\section{Author details}

${ }^{1}$ Department of Nephrology, Ruijin Hospital, Shanghai Jiao Tong University School of Medicine, 197 Ruijin Er Road, Shanghai 200025, People's Republic of China. ${ }^{2}$ Department of General Surgery, Ruijin Hospital, Shanghai Jiao Tong University School of Medicine, Shanghai 200025, People's Republic of China.

${ }^{3}$ Department of Radiology, Ruijin Hospital, Shanghai Jiao Tong University School of Medicine, Shanghai 200025, People's Republic of China.

Received: 27 November 2019 Accepted: 27 February 2020

Published online: 05 March 2020

\section{References}

1. Davidson A. What is damaging the kidney in lupus nephritis? Nat Rev Rheumatol. 2016;12(3):143-53.

2. Laurent $\mathrm{D}$, Helene $\mathrm{S}$, Roch $\mathrm{G}$, et al. Tubular lesions and tubular cell adhesion molecules for the prognosis of lupus nephritis. Kidney Int. 1994;45:1285-300.

3. Nakatani $\mathrm{K}$, Fujii $\mathrm{H}$, Hasegawa $\mathrm{H}$, et al. Endothelial adhesion molecules in glomerular lesions: association with their severity and diversity in lupus models. Kidney Int. 2004;65(4):1290-300.

4. Polanowska-Grabowska R, Wallace K, Field JJ, et al. P-selectin-mediated platelet-neutrophil aggregate formation activates neutrophils in mouse and human sickle cell disease. Arterioscler Thromb Vasc Biol. 2010;30(12):2392-9.

5. Morariu AM, Schuurs TA, Leuvenink HG, et al. Early events in kidney donation: progression of endothelial activation, oxidative stress and tubular injury after brain death. Am J Transplant. 2008;8(5):933-41. 
6. Fuller TF, Sattler B, Binder L, Vetterlein F, Ringe B, Lorf T. Reduction of severe ischemia/reperfusion injury in rat kidney grafts by a soluble P-selectin glycoprotein ligand. Transplantation. 2001;72(2):216-22.

7. Herget-Rosenthal S. Imaging techniques in the management of chronic kidney disease: current developments and future perspectives. Semin Nephrol. 2011;31(3):283-90.

8. Xu X, Fang W, Ling H, Chai W, Chen K. Diffusion-weighted MR imaging of kidneys in patients with chronic kidney disease: initial study. Eur Radiol. 2010;20(4):978-83.

9. Wang W, Yu Y, Wen J, et al. Combination of functional magnetic resonance imaging and histopathologic analysis to evaluate interstitial fibrosis in kidney allografts. Clin J Am Soc Nephrol. 2019;14(9):1372-80.

10. Pedersen M, Dissing TH, Mørkenborg J, et al. Validation of quantitative BOLD MRI measurements in kidney: application to unilateral ureteral obstruction. Kidney Int. 2005;67(6):2305-12.

11. Li X, Xu X, Zhang Q, Ren H, Zhang W, Liu Y, Yan F, Chen N. Diffusion weighted imaging and blood oxygen level-dependent mr imaging of kidneys in patients with lupus nephritis. J Transl Med. 2014;12:295.

12. Rapacchi S, Smith RX, Wang Y, Yan L, Sigalov V, Krasileva KE, Karpouzas G, Plotnik A, Sayre J, Hernandez E, Verma A, Burkly L, Wisniacki N, Torrington J, He X, Hu P, Chiao PC, Wang DJ. Towards the identification of multiparametric quantitative mri biomarkers in lupus nephritis. Magn Reson Imaging. 2015;33:1066-74.

13. Yu F, Wu LH, Tan Y, et al. Tubulointerstitial lesions of patients with lupus nephritis classified by the 2003 International Society of Nephrology and Renal Pathology Society system. Kidney Int. 2010;77(9):820-9.

14. Brezis M, Rosen S. Hypoxia of the renal medulla-its implications for disease. N Engl J Med. 1995;332(10):647-55.

15. Palm F, Nordquist L. Renal tubulointerstitial hypoxia: cause and consequence of kidney dysfunction. Clin Exp Pharmacol Physiol. 2011;38(7):474-80.

16. Millar TM, Phan V, Tibbles LA. ROS generation in endothelial hypoxia and reoxygenation stimulates MAP kinase signaling and kinase-dependent neutrophil recruitment. Free Radic Biol Med. 2007;42(8):1165-77.

17. Simon $N$, Hertig A. Alteration of fatty acid oxidation in tubular epithelial cells: from acute kidney injury to renal fibrogenesis. Front Med (Lausanne). 2015;2:52.

18. Passwell J, Schreiner GF, Nonaka M, Beuscher HU, Colten HR. Local extrahepatic expression of complement genes C3, factor B, C2, and C4 is increased in murine lupus nephritis. J Clin Invest. 1988;82(5):1676-84.

19. Du Y, Sanam S, Kate K, Mohan C. Animal models of lupus and lupus nephritis. Curr Pharm Des. 2015:21(18):2320-49.

20. Theofilopoulos AN, Dixon FJ. Murine models of systemic lupus erythematosus. Adv Immunol. 1985;37:269-390.
21. Harjunpää H, Llort Asens M, Guenther C, Fagerholm SC. Cell adhesion molecules and their roles and regulation in the immune and tumor microenvironment. Front Immunol. 2019;10:1078.

22. Dole VS, Bergmeier W, Mitchell HA, et al. Activated platelets induce Weibel_-Palade-body secretion and leukocyte rolling in vivot role of P-selectin. Blood. 2005;106(7):2334-9.

23. Li X, Zhou T, Hao C, et al. Significance of P-selectin expression in glomerulonephritis. Chin Med J. 1997;110(7):512-4.

24. Ghoshal P, Rajendran M, Odo N, et al. Glycosylation inhibitors efficiently inhibit P-selectin-mediated cell adhesion to endothelial cells. PLOS ONE. 2014;9(6):e99363.

25. Ley K. The role of selectins in inflammation and disease. Trends Mol Med. 2003:9(6):263-8.

26. Morris DL, Graham RR, Erwig LP. Variation in the upstream region of P-selectin (SELP) is a risk factor for SLE. Genes Immun. 2009;10(5):404-13.

27. Li X, Zhou T, Sun GZ, et al. Clinical significance of dendritic cells distribution in renal tissues of lupus nephritis. J Modern Immun. 2004;24(2):142-5.

28. He X, Schoeb TR, Panoskaltsis-Mortari A, et al. Deficiency of P-selectin or P-selectin glycoprotein ligand-1 leads to accelerated development of glomerulonephritis and increased expression of CC chemokine ligand 2 in lupus-prone mice. J Immunol. 2006;177(12):8748-56.

29. Brezis M, Rosen S. Hypoxia of the renal medulla - its implications for disease. N Eng J Med. 1995;332:647-55.

30. Palm F, Nordquist I. Renal tubulointerstitial hypoxia: cause and consquence of kidney dysfunction. Clin Exp Pharmacol Physiol. 2011:38:424-30.

31. Stroka DM, Burkhardt T, Desbaillets I, et al. HIF-1 is expressed in normoxic tissue and displays an organ-specific regulation under systemic hypoxia. FASEB J. 2001;15:2445-53.

32. Hopfl G, Ogunshola O, Gassmann M. HIFs and tumors-causes and consquences. Am J Physiol Regul Integr Comp Physiol. 2004;286:R608-23.

33. Tardif JC, Tanguay JF, Wright SR, et al. Effects of the P-selectin antagonist inclacumab on myocardial damage after percutaneous coronary intervention for non-ST-segment elevation myocardial infarction: results of the SELECT-ACS trial. J Am Coll Cardiol. 2013;61(20):2048-55.

34. Stähli BE, Tardif JC, Carrier M, et al. Effects of P-selectin antagonist inclacumab in patients undergoing coronary artery bypass graft surgery: SELECT-CABG Trial. J Am Coll Cardiol. 2016;67(3):344-6.

\section{Publisher's Note}

Springer Nature remains neutral with regard to jurisdictional claims in published maps and institutional affiliations.
Ready to submit your research? Choose BMC and benefit from:

- fast, convenient online submission

- thorough peer review by experienced researchers in your field

- rapid publication on acceptance

- support for research data, including large and complex data types

- gold Open Access which fosters wider collaboration and increased citations

- maximum visibility for your research: over $100 \mathrm{M}$ website views per year

At BMC, research is always in progress.

Learn more biomedcentral.com/submissions 